\title{
La búsqueda del lector híbrido como motivo implícito de la traducción pragmática. Caso de la novela Puppet de Margarita Cota-Cárdenas y su traducción al inglés
}

\author{
The search of a hybrid reader as an implicit motive for \\ pragmatic translation. The case of the novel Puppet by \\ Margarita Cota-Cárdenas \\ and its translation into English
}

\author{
Iwona Kasperska \\ Uniwersytet im. Adama Mickiewicza w Poznaniu (Polonia) \\ iwona.kasperska@amu.edu.pl
}

\begin{abstract}
The article deals with a particular case of intra- and intertextual translation of a highly pragmatic nature, which can be seen in Puppet. A Chicano novella by Margarita Cota-Cárdenas. Composed mainly of fragments of conversations, monologues and personal letters, the novel reflects Spanish-English codeswitching, as a characteristic feature of the speech of the Mexican American community in the USA. By (re)producing a range of heteroglossal forms of this community communication, Cota-Cárdenas uses translation procedures to reach the hybrid reader who is, at the same time, the hallmark of Chicanos. The multilingual strategies (Hansen Esplin, 2012) of the author and the translators, Barbara D. Riess and Tino Sandoval, become the main pragmatic objective of the novel, both in its source and target versions. Given the multitude of translation procedures observed in both versions of this hybrid novel, the relativity of traditional translation terms, such as original and translation, is also discussed, as both turn out to be bilingual texts and pragmatic translations.
\end{abstract}

Keywords: Chicano/a literature, Margarita Cota-Cárdenas's Puppet, hybrid text, pragmatic translation, hybrid reader 


\section{INTRODUCCIÓN}

El presente artículo trata de un caso particular de la traducción intra- e intertextual de carácter altamente pragmático, que se puede observar en dos versiones de la novela Puppet de Margarita Cota-Cárdenas. Nos encontramos ante un caso especial de texto híbrido, tanto desde el punto de vista lingüístico como genérico, y con su traducción híbrida al inglés, efectuada por Barbara D. Riess y Tino Sandoval, en colaboración con la autora. Aparte de los rasgos característicos mencionados, merece la pena subrayar que es un texto emblemático del chicanismo comprometido, que coloca en primer plano el tema de la identidad lingüística y cultural de la comunidad de Mexican Americans en los Estados Unidos de América del Norte. Estas dos identidades resultan ser esenciales e inseparables, lo cual se verá en el siguiente estudio donde analizaré los procedimientos traductológicos que se propusieron realizar la autora y los traductores para alcanzar sus metas.

En primer lugar, presentaré brevemente la literatura chicana, resaltando los rasgos característicos de la novela Puppet, tanto los que la hacen inscribirse plenamente en dicha corriente como los que la destacan como innovadora y experimental. Después me enfocaré en la problemática lingüística de la literatura chicana, haciendo hincapié en la(s) lengua(s) en la(s) que se escribe y las implicaciones políticas y culturales de las decisiones tomadas al respecto por los autores y las editoriales. Intentaré, a su vez, responder a la pregunta de a qué tipo de lector se dirige esta literatura. En cuanto a la novela Puppet, me interesará el texto de partida (en adelante: TP) escrito en español y las técnicas de traducción intratextual, así como el texto meta (en adelante: TM) en inglés y las técnicas de traducción intertextual. Los procedimientos examinados me permitirán reflexionar sobre los objetivos pragmáticos que querían alcanzar la autora y los traductores.

\section{PUPPET COMO UNO DE LOS TEXTOS EMBLEMÁTICOS DE LA LITERATURA CHICANA}

La literatura chicana, en el sentido más restringido de la palabra, está relacionada con el ideario del Movimiento Chicano por los Derechos Civiles, que surgió en los Estados Unidos a mediados de los años 60 del siglo XX (Kaganiec-Kamieńska, 2008; Gonzales, 2009). La primera década de su desarrollo se ha denominado como el "Florecimiento" o el "Renacimiento", dadas la proliferación y la temática de la producción literaria, que fue una reacción a la necesidad histórica de debatir sobre la situación de la comunidad mexicana en los Estados Unidos (Lomelí, 2002). En su sentido más amplio, se trata de la literatura escrita por los autores americanos de ascendencia mexicana, asimilados en distintos grados a la cultura anglosajona, que se identifican con sus raíces mexicanas y adoptan la actitud de compromiso social con su comunidad (Villanueva, 1980). Los críticos literarios clasifican esta literatu- 
ra como mexicano-americana o literatura menor (Rudin, 1996), hispano-americana (López Ponz, 2014) o Latino/a literature (Bost, Aparicio, 2013), entre muchos otros términos. La mayoría de estas denominaciones sugiere el carácter bilingüe de los textos, dado el contacto lingüístico que se produjo una vez cruzada la frontera mexicano-americana por distintas generaciones de mexicanos. Sin duda alguna, vemos aquí escritura híbrida donde lo formal destaca la compleja identidad lingüístico-cultural de los chicanos, que en muchas ocasiones ha sido/es el resultado del choque cultural y la falta de compatibilidad entre la cultura de origen y la de adopción.

La autora de Puppet, Margarita Cota-Cárdenas, pertenece a la generación de escritoras ${ }^{1}$ cuya obra contribuyó a la innegable explosión de la literatura chicana femenina en las décadas de 1980 y 1990 (Lomelí, Márquez, Herrera-Sobek, 2000), llegando incluso a denominarse "Década de la Chicana". Las autoras de dicha generación se ocuparon de la temática chicana general, a saber: la experiencia de los (in)migrantes mexicanos en los Estados Unidos, las etapas de la compleja y, a veces, traumática asimilación, la actitud frente a la cultura anglosajona del país receptor, la mitología mexicana, el sistema de valores y la cultura material de la comunidad de Mexican Americans. No obstante, en la escritura femenina destacan nuevos enfoques posmodernos, como un mayor interés por una perspectiva muy personal y más vertical que horizontal, así como la preferencia por la hibridez de formas genéricas y lingüísticas (Lomelí et al., 2000). Las chicanas debaten en ella sobre la opresión de género, raza y clase, revisan las narraciones fundamentales de la mexicanidad, la "doble identidad" cultural, el papel de los personajes mitológicos e históricos como la Virgen de Guadalpe, la Llorona y Malinche, narran su experiencia personal como forma de expresar su etnicidad (Zygadło, 2007).

En cuanto a la novela Puppet, su publicación en 1985 es considerada un verdadero parteaguas para la literatura chicana femenina. En primer lugar, el texto fue declarado como "escrito en español" y, en segundo lugar, fue concebido por una mujer, además de ser temática y formalmente controvertido. Puppet es una novela ambientada en el contexto del Movimiento Chicano. La acción abarca casi dos decenios y tiene como fondo los cambios sociales y culturales que se producen en los EE.UU. durante la guerra de Vietnam, la lucha de la comunidad afroamericana por los derechos civiles y el feminismo cada vez más activo (Rebolledo, 2005). La protagonista, Petra Leyva, trabaja primero como secretaria en la empresa constructora Southwest City Estates y, posteriormente, como profesora de español en una universidad. Adquiere cada vez más conciencia chicana y feminista, lo cual pone en riesgo la relación con su propia familia, que es tradicional y tiene muy claro cuál es el papel de la mujer en la vida y dentro de la familia. El título de la novela es el seudónimo de un joven mexicano de diecisiete años, Tony López, que trabaja en la misma empresa que Petra. El muchacho encuentra su trágica muerte a manos de la policía, que quiere encubrir el injusto asesinato.

\footnotetext{
${ }^{1}$ Junto con Denise Chávez, Helena María Viramontes, Sandra Cisneros, Pat Mora y Erlinda Gonzales-Berry (Rebolledo, 2000).
} 
Puppet es una novela experimental, vanguardista y posmoderna que resalta la discontinuidad de la conciencia por medio de voces, fragmentos de conversaciones y microrrelatos que hacen muy difícil cualquier intento de delimitar el eje narrativo principal (Manzanas Calvo, 2012). La obra compagina elementos de la novela detectivesca, social, histórica y política, a los que acompaña un ambiente de ambigüedad, paranoia y opresión (Rebolledo, 2005).

\section{LA FORMAS DE HABLAR DE LOS CHICANOS}

Las múltiples formas de expresión de los chicanos son clasificadas por los lingüistas como bilingüismo (multilingüismo), codeswitching, codemixing, spanglish o interlengua. Todos estos términos se complementan, poniendo énfasis en uno u otro aspecto lingüístico de la comunicación y en cada caso siendo una manifestación de la doble identidad.

El bilingüismo consiste en hablar en dos (y el multilingüismo en dos o más) lenguas (o dialectos) en la vida diaria. Dichas lenguas pueden ser usadas en distintas configuraciones, según las preferencias y necesidades comunicativas individuales del locutor (Grosjean, 2010). Todos los chicanos pueden ser considerados bilingües (o multilingües) porque usan varios códigos que dominan en distintos grados (Peñalosa, 1975).

En cuanto al codeswitching (cambio de código o alternancia de lenguas), este consiste en recurrir a dos lenguas (o dialectos), a nivel interoracional. En cambio, el codemixing (mezcla de códigos) consiste en usar dos lenguas (o dialectos) a nivel intraoracional. Desde el punto de vista pragmático, el codeswitching ocurre mientras el locutor está eligiendo la lengua (función pragmática situacional) y consiste en resaltar el énfasis y el contraste (función pragmática metafórica). El codeswitching desempeña también muchas funciones estilístico-pragmáticas, a saber: las interjecciones, las expresiones idiomáticas, las coletillas y las citas (indicadas por Poplack); los paréntesis, el énfasis, las exclamaciones, las repeticiones, las alternaciones simétricas, las frases rutinarias y la anticipación, aparte del léxico aleatorio, el léxico requerido, así como las unidades léxicas desencadenantes, el cambio de estilo y los marcadores discursivos, observados por Valdés Fallis (Montes-Alcalá, 2001). A todas luces, nos topamos con una colección abundante de objetivos pragmáticos del codeswitching y el codemixing.

Los lingüistas hablan también del spanglish, una fusión del español y el inglés, típica de la comunidad hispana en los Estados Unidos, sin distinguir la clase social. López García-Molins (2015) considera el spanglish como "un dialecto del español, que consiste en un cruce neurolingüístico que se traduce en la inserción de anglicismos en estructuras gramaticales españolas" (pp. 99-100). Sostiene además que "no se trata de un dialecto espacial, social o pragmático, sino de un dialecto psicológico": los hispanos en los Estados Unidos, como bilingües constitutivos (o compuestos), "tienen 
asociadas cerebralmente en una red sináptica permanente las dos versiones de cualquier elemento lingüístico" (López García-Molins, 2016, p. 105).

Entre dos polos lingüísticos constituidos por el español estándar y el inglés estándar, se encuentra un sinfín de mezclas de ambos idiomas, incluidas sus variantes dialectales y sociolectos (Bruce-Novoa, 1980). El lenguaje de los chicanos es "a blend, a synthesis of the two into a third. Thus they are interlingual, not bilingual. The codes are not separate, but intrinsically fused" (p. 29). De esta descripción se desprende que la mezcla del inglés y el español se convierte en "un tercer" código que Bruce-Novoa $(1980,1990)$ llama "interlengua" o "idioma personal". En las letras chicanas los autores elaboran este código creativa y artísticamente, sirviéndose de la fusión gramatical y sintáctica castellano-inglesa, así como de las alusiones transculturales. La interlengua chicana es una práctica lingüística muy dependiente del contexto, por lo cual es espontánea e irrepetible.

\section{4. ¿ESPAÑOL O INGLÉS? ESA ES LA CUESTIÓN}

En cuanto al uso del español y el inglés, en primer lugar hay que subrayar que no todos los autores chicanos son capaces de manejar ambos idiomas para crear textos artísticos. La elección de la lengua inglesa es, entonces, dictada por las circunstancias personales. La respuesta a la pregunta sobre en qué lengua(s) escribir/editar la literatura "menor", está directamente relacionada con la economía (el número de lectores potenciales que van a comprar el libro) y la política (¿es la literatura un espacio adecuado para reivindicar y visibilizar la lengua de herencia?).

La lengua dominante en la que las editoriales publican la literatura chicana es el inglés. Antes del Florecimiento chicano, cuando el interés por parte de los lectores y las editoriales era mínimo, fueron fundadas pequeñas editoriales enfocadas en un público específico. Merece la pena precisar que el carácter político de la elección de la lengua tiene sus límites: son tan solo los autores realmente bilingües los que son capaces de crear textos artísticos en ambos idiomas (Bruce-Novoa, 1990). Sin embargo, cuando la lengua española perdió el interés de los lectores que se asimilaron y empezaron a leer textos en inglés, la principal editorial chicana Quinto Sol de Berkeley, por ejemplo, inició las publicaciones bilingües de textos en español, es decir, con una traducción al inglés, mientras que editaba los textos en inglés sin traducción al castellano. Martín-Rodríguez (2003) ha introducido la distinción entre "the market" (el mercado anglo del establishment) y "la marketa" (el mercado hispano de la comunidad chicana), recalcando que las decisiones sobre la lengua de publicación son tomadas en función del uno o la otra. La literatura chicana, gracias a su forma multilingüe, va dirigida a un público que tiene el mismo perfil, es decir, que también es multilingüe.

Cuando Cota-Cárdenas estaba escribiendo Puppet en los años 80, las cuestiones relacionadas con la lengua se encontraban en el centro del debate entre los literatos 
chicanos que "luchaban con los complejos de representación, identidad, subjetividad, temática e ideología" (Rebolledo, 2000, p. xiii). Al preparar la novela, la autora decidió concebirla en español, justificando su decisión por el deseo de incluir en su texto giros específicos que se grabaron en español en su memoria, como bromas o dichos, sin traducirlos al inglés. Por encima de todo, era su "resistance to what she felt was linguistic and cultural annihilation" (p. xiv).

\section{EL MULTILINGÜISMO EN LA LITERATURA CHICANA: TRADUCCIÓN INTRATEXTUAL EN PUPPET}

La lectura de Puppet evidencia su carácter bilingüe y un repertorio muy amplio de recursos traductológicos que se usan a nivel del TP. Como en este estudio me interesan los objetivos pragmáticos de los procedimientos aplicados, planteo las siguientes preguntas: 1) ¿Qué forma tienen los incisos ingleses en el TP? 2) ¿Se traducen al español o se quedan sin traducción? 3) Si se traducen, ¿qué procedimientos de traducción son aplicados? 4) ¿Puede un lector monolingüe hispano o anglófono leer el texto de la "misma manera"?

El análisis efectuado ha revelado que los incisos en inglés incluidos en Puppet representan un amplio abanico de formas. Entre ellas se pueden distinguir los incisos sencillos, como palabras sueltas y sintagmas, que desempeñan distintas funciones semánticas y pragmáticas, a saber: invocaciones (my God), saludos (hello), palabras culturales relacionadas con la realidad americana (welfare), adverbios (yes, no) y marcadores del discurso (well, look), vocabulario general y especializado (builders, District Attorney), topónimos (Southwest City) y oraciones sencillas (I know). La posición que dichos incisos ocupan dentro de la oración, el microcontexto semántico y el codemixing permiten descifrar su significado. Por ejemplo: "vestido en sweatshirt", "darle polish a los pisos de madera".

Otros incisos ingleses se deben al cambio de código. Dada la fragmentariedad del texto y su carácter oral, es fácil observar el idiolecto de los personajes que representan variados perfiles étnicos, sociales y culturales. Desde luego, las más interesantes resultan las réplicas de los chicanos porque permiten descifrar la riqueza de códigos que usan y su carácter pragmático. Petra Leyva, por ejemplo, se autodefine como chicana pero su circunstancia de pertenecer a la segunda generación de mexicanos la "condena" al constante vaivén entre ambas lenguas y culturas de su identidad (verbigracia, usa hasta tres formas de su nombre). Significa también la imposibilidad de elegir una de ellas de una vez por todas, como en el siguiente ejemplo:

—...Aló, I mean Good Afternoon, Southwest City Estates... No, Stan isn’t here... Who?... Oh, Paco Jiménez from the Mex-Am C of C... Ajá... sí, hablo esp... mande...? No, no creo que nos hayamos conocido... no, en ningún baile... ni quinceañera (jiji)... What can 
I do for you Mr... oh, Paco, all right... Boletos...? You want me to ask Stan if he'd buy two boletos for the Fiestas Patrias Cotillion next week... ajá, sí, cómo no... I'll leave the message for him... Well, it's almost five o'clock and he's not back from the Title Company... Sure... cómo no, hasta luego... Excuse-me? Oh!... Petra, Patricia Leyva de... well, just Pat Leyva is fine... Sure, no trouble... bye... (Cota-Cárdenas, 2000, p. 100, énfasis de la autora)

En esta conversación telefónica, en la que escuchamos solo a Petra, es notable su afán de adaptarse al interlocutor que, probablemente, también es Mexican American (solo siendo mexicanos pueden conocer la tradición de quinceañera o estar interesados en las Fiestas Patrias). En el habla bilingüe de Petra observamos la constante alternancia de códigos: el español estándar (con el vocabulario típico de la variante mexicana, como boletos, mande, cómo no) y el inglés estándar. En su idiolecto son muy características la combinación hispano-inglesa "Fiestas Patrias Cotillion" y la asociación de dos versiones de un solo elemento lingüístico en "sure/cómo no". En los enunciados de Petra domina la lengua inglesa debido al hecho de que se trata de una conversación oficial y, en realidad, son los incisos españoles los que fueron infiltrados en la lengua inglesa que quiere manejar la protagonista.

El cambio de código y la mezcla de códigos contribuyen a darle el aspecto bilingüe a la novela, lo cual fue destacado por algunos críticos (Rudin, 1996; Hansen Esplin, 2012). Ciertos pasajes están escritos únicamente en inglés (cuando se trata de americanos monolingües), por lo cual son incomprensibles para un lector hispano monolingüe. Este recurso da la impresión de que la autora no da concesión a los lectores monolingües y monoculturales. A quien da preferencia es a un receptor tan híbrido como los chicanos u otras comunidades de los Hispanics cuyo perfil lingüístico-cultural se asemeja al de los Mexican Americans. Puppet tiene una construcción pragmática en el sentido de que su objetivo básico es reflejar los usos lingüísticos reales, sin que importen las "carencias" del lector no híbrido.

Ahora bien, al revisar el texto según el criterio de traducción y comprensión de los incisos multilingües, se pueden observar los procedimientos incluidos en la Tabla 1.

Tabla 1. Procedimientos de traducción intratextual en la novela Puppet (Elaboración propia)

\begin{tabular}{|l|l|}
\hline \multicolumn{1}{|c|}{ Puppet como texto de partida escrito en español } & \multicolumn{1}{c|}{ Procedimientos de traducción } \\
\hline Incisos sencillos en inglés & $\begin{array}{l}\text { No traducción } \\
\text { Codemixing }\end{array}$ \\
\hline Incisos largos en inglés & $\begin{array}{l}\text { No traducción } \\
\text { Traducción libre yuxtapuesta } \\
\text { Codeswitching } \\
\text { Codemixing }\end{array}$ \\
\hline $\begin{array}{l}\text { Incisos sencillos y largos en el dialecto del español ha- } \\
\text { blado en el Southwest y el Norte de México }\end{array}$ & \\
\hline
\end{tabular}


Hansen Esplin (2016) pone énfasis en el hecho de que Cota-Cárdenas no acomoda el texto de Puppet a los lectores monolingües. La autora misma parece darse cuenta de la dificultad de la lectura debido a las "acrobacias lingüísticas" con las que quiere reforzar sus declaraciones teóricas de corte lingüístico, cultural e identitario, proponiendo un texto multilingüe que representa a todas las comunidades hispanas del Southwest. No en balde, novelas como Puppet fueron clasificadas como "half-breed writing" (Manzanas Calvo, 2012, p. 48).

\section{TRADUCCIÓN DE TEXTOS CHICANOS: TRADUCCIÓN INTERTEXTUAL DE PUPPET}

En el estudio contrastivo de Puppet con su versión inglesa me interesan las siguientes cuestiones: 1) ¿Cuál es la estrategia general de traducir el TP híbrido? 2) ¿Qué procedimientos de traducción pragmática son aplicados para reflejar el lenguaje de los chicanos? 3) ¿Qué grado de dificultad representa el TM para un lector anglófono monolingüe? 4) ¿Hay diferencias entre los procedimientos de traducción usados en el TP y en el TM? El análisis contrastivo ha desvelado la restitución del codeswitching hispano-inglés en el TM, pero con la reversión de las proporciones de ambas lenguas. Los traductores recurren a una serie de procedimientos para reflejar también la hibridez del habla chicana. Para aclarar dicha estrategia de carácter pragmático, veamos el siguiente ejemplo:

\begin{tabular}{|c|c|}
\hline $\begin{array}{c}\text { Puppet } \\
\text { TP }\end{array}$ & $\begin{array}{c}\text { Puppet } \\
\text { TM }\end{array}$ \\
\hline $\begin{array}{l}\text {-Pat, no puedo vinir al trabajo mañana por la } \\
\text { mañana... No, es que Félix tiene que ir a la corte } \\
\text { por un hearing... Abogado? Que quién lo repre- } \\
\text { senta? Pues, pues tiníanos abogado pero pues es } \\
\text { miedoso... someone got to him or something, nos } \\
\text { llamó que buscáranos a jotro, y como no hay quie- } \\
\text { nes sean raza y es al último momento, pos tengo } \\
\text { yo que ir a... pues tú sabes, a ver qué se arregla... } \\
\text { (104, énfasis míos) }\end{array}$ & $\begin{array}{l}\text { "Pat, I can't come to work tomorrow morning... } \\
\text { No, is just that Félix has to go to court for a hea- } \\
\text { ring... Lawyer? Who represents him? Pues, pues } \\
\text { we had a lawyer but he's afraid... someone got to } \\
\text { him or something, he called and told us to look for } \\
\text { another one and since there isn't one who is raza } \\
\text { and it's at the last minute, pos I have to go to... } \\
\text { pues tú sabes, you know, we'll see what can be } \\
\text { done..." (111, énfasis míos) }\end{array}$ \\
\hline
\end{tabular}

En la réplica de Memo, otro personaje chicano, se notan formas dialectales, como vinir, tiníanos, buscáranos y jotro, normalizadas en el TM. Solo el marcador discursivo pos, en su forma coloquial, fue transferido a la versión inglesa. En el léxico de Memo aparece un lexema especializado, hearing, y la frase someone got to him or something, cuya función de incisos ingleses desaparece naturalmente en el TM. También es transferida la palabra cultural raza, que se refiere a la comunidad de mexicanos. Finalmente, vemos que los traductores aprovechan la alternación simétrica de códigos como procedimiento de traducción yuxtapuesta. 
Desde luego se trata tan solo de una muestra de los procedimintos aplicados en esta estrategia de traducción pragmática que refleja la hibridez lingüística de la comunidad chicana. El TM reproduce el cambio y la mezcla de códigos, pero con la dominación de la lengua inglesa. La alternancia consiste en anteponer o en posponer la unidad traducida al inglés por medio de un equivalente, una traducción literal o libre, así como por medio de una explicación. En lo que se refiere a la comprensión del texto por el lector anglófono monolingüe, el TM puede ser más fácil para este, que la versión española para el lector hispanófono monolingüe: en términos de cantidad, el TM contiene más lengua inglesa que el TP tiene de lengua española. La edición bilingüe, usada en este análisis, se presta entonces para una lectura simultánea o alternativa para los lectores monolingües.

Para poder comparar los recursos de traducción en el TP y el TM, propongo la siguiente tabla:

Tabla 2. Procedimientos de traducción en Puppet (Elaboración propia)

\begin{tabular}{|l|l|l|}
\hline \multicolumn{2}{|c|}{$\begin{array}{c}\text { Puppet, } \\
\text { traducción intratextual }\end{array}$} & \multicolumn{1}{c|}{$\begin{array}{c}\text { Puppet, } \\
\text { traducción intertextual }\end{array}$} \\
\hline \multicolumn{2}{|c|}{ TP } & \multicolumn{1}{c|}{ TP } \\
\hline Incisos sencillos en inglés & $\begin{array}{l}\text { No traducción } \\
\text { Codemixing }\end{array}$ & $\begin{array}{l}\text { Incisos sencillos y pasajes largos } \\
\text { en inglés }\end{array}$ \\
\hline Incisos largos en inglés & $\begin{array}{l}\text { No traducción } \\
\text { Traducción libre yuxtapuesta } \\
\text { Codeswitching } \\
\text { Codemixing }\end{array}$ & Traducción español-inglés \\
\hline $\begin{array}{l}\text { Incisos sencillos y largos en el } \\
\text { dialecto del español hablado en } \\
\text { el Southwest }\end{array}$ & $\begin{array}{l}\text { Incisos sencillos y largos en el } \\
\text { dialecto del español hablado en } \\
\text { el Southwest }\end{array}$ \\
\hline
\end{tabular}

La Tabla 2 refleja una gama de procedimientos de traducción tanto en el TP como en el TM. Prácticamente, hallamos el mismo repertorio de procedimientos, excepto la transferencia de los incisos ingleses al TM inglés. En el TP, la lengua española es representada por la variante estándar y por el dialecto norteño del español, lo cual hace esta lista más larga. El cotejo de los textos evidencia que ambos son híbridos y chicanos. La diferencia entre ellos consiste en la distinta proporción del español y el inglés, incluidos los giros y léxicos dialectales. Por consiguiente, la configuración de códigos en el TM favorece al lector anglófono, sin dejar de ser un texto híbrido. Un procedimiento pragmático más es que la versión en inglés, con los nombres de los traductores expuestos en la portada, viene como primera en la edición bilingüe. De ahí mi conclusión de que se favorezca, de hecho, al lector anglófono mientras el TP se encuentra del otro lado del libro. 
Tanto la hibridez lingüística de ambos textos como el revertido orden tradicional de original-traducción en la "edición bilingüe", relativizan la obsoleta terminología traductológica. De hecho, sin la portada, sería muy difícil distinguir entre el TP y el TM.

\section{CONCLUSIÓN}

La edición "monolingüe" de Puppet (1985) se quedó en "virtual obscuridad", mientras que la bilingüe obtuvo un "relativo éxito" (Hansen Esplin, 2012, p. 25). Estas calificaciones hacen pensar en un valor añadido al proyecto editorial del año 2000, sometido al anális. La edición sinóptica con la introducción en inglés de Tey Diana Rebolledo resalta el carácter altamente pragmático de la obra de Cota-Cárdenas y la hacen un poco más accesible a todo tipo de lectores. Los usos lingüísticos reales y la diversidad de códigos usados en el Southwest estadounidense aumentan la dimensión identitaria de la novela. Como texto chicano mestizo, por ser "un continuo trasvase entre dos, tres o más lenguas" (López Ponz, 2009, p. 57), la novela Puppet ya es una traducción pragmática de la hibridez chicana. 


\section{BIBLIOGRAFÍA}

Bost, S., Aparicio, F.R. (Eds.) (2013). The Routledge Companion to Latino/a Literature. London-New York: Routledge.

Bruce-Novoa, J. (1980). Chicano Authors. Inquiry by Interview. Austin-London: University of Texas Press. Bruce-Novoa, J. (1990). RetroSpace: Collected Essays on Chicano Literature. Houston: Arte Público Press. Cota-Cárdenas, M. (2000). Puppet. Una novella chicana/Puppet. A Chicano novella. Albuquerque, NM: University of New Mexico Press.

Gonzales, M.G. (2009). Mexicanos. A History of Mexicans in the United States. Bloomington, IN: Indiana University Press.

Grosjean, F. (2010). Bilingual. Life and Reality. Cambridge-London: Harvard University Press.

Hansen Esplin, M. (2012). Spanish, English, and in-between: self-translation in the U.S. and Latin America. Recuperado de: https://d.lib.msu.edu/etd/768.

Hansen Esplin, M. (2016). Self-translation and Accommodation: Strategies of Multilingualism in Gloria Anzaldúa's Borderlands/La Frontera: The New Mestiza and Margarita Cota-Cárdenas's Puppet. MELUS, 41 (2), 176-201. DOI: 10.1093/melus/mlw012.

Kaganiec-Kamieńska, A. (2008). Tożsamość na pograniczu kultur. Meksykańska grupa etniczna w Stanach Zjednoczonych. Kraków: UNIVERSITAS.

Lomelí, F.A. (2002). An Interpretative Assessment of Chicano Literature and Criticism. In J. Benito \& A.M. Manzanas (Eds.), Literature and Ethnicity in the Cultural Borderlands (pp. 63-79). Amsterdam-New York: Rodopi.

Lomelí, F.A., Márquez, T., Herrera-Sobek, M. (2000). Trends and Themes in Chicano/a Writings in Postmodern Times. In D.R. Maciel, I.D. Ortiz \& M. Herrera-Sobek (Eds.), Chicano Renaissance. Contemporary Cultural Trends (pp. 285-312). Tucson: The University of Arizona Press.

López García-Molins, A. (2015). Teoría del spanglish. Valencia: Tirant Humanidades.

López García-Molins, A. (2016). El spanglish como dialecto psicológico. In S. Betti \& E. Serra Alegre (Eds.), Nuevas voces sobre el spanglish. Una investigación polifónica (pp. 105-116). New York: ANLE.

López Ponz, M. (2009). Traducción y literatura chicana: nuevas perspectivas desde la hibridación. Granada: Editorial Comares.

López Ponz, M. (2014). Juegos de capitales. La traducción en la sociedad del mestizaje. Frankfurt am Main: Peter Lang.

Manzanas Calvo, A.M. (2012). A Mestiza in the Borderlands: Margarita Cota-Cárdenas' Puppet. Atlantis. Journal of the Spanish Association of Anglo-American Studies, 34 (1), 47-62.

Martín-Rodríguez, M.M. (2003). Life in Search of Readers: Reading (in) Chicano/a Literature. Albuquerque, NM: University of New Mexico Press.

Montes-Alcalá, C. (2001). Written codeswitching: Powerful bilingual images. In R. Jacobson (Ed.), Codeswitching Worldwide II (pp. 193-219). Berlin-New York: Mouton de Gruyter.

Peñalosa, F. (1975). Chicano Multilingualism and Multiglossia. In E. Hernández-Chávez, A. D. Cohen \& A.F. Beltramo (Eds.), El Lenguaje de los Chicanos. Regional and Social Characteristics Used by Mexican Americans (pp. 164-169). Arlington, VA: Center for Applied Linguistics.

Rebolledo, T.D. (2000). Foreword. In M. Cota-Cárdenas, Puppet. Una novella chicana/ Puppet. A Chicano novella (pp. xiii-xxii). Albuquerque, NM: University of New Mexico Press.

Rebolledo, T.D. (2005). Creating Sanctuaries of the Heart: An Introduction. In M. Cota-Cárdenas, Sanctuaries of the Heart/Santuarios del Corazón (pp. ix-xvii). Tucson: The University of Arizona Press.

Rudin, E. (1996). Tender accents of sound. Spanish in the Chicano Novel in English. Tempe, AZ: Bilingual Press/Editorial Bilingüe.

Villanueva, T. (1980). Prólogo. Sobre el término “chicano". In T. Villanueva (Comp.), Chicanos. Antología histórica y literaria (pp. 7-67). México: FCE.

Zygadło, G. (2007). Culture Matters. Chicanas'Identity in Contemporary USA. Frankfurt am Main: Peter Lang. 